\title{
HEREDITARY LOCAL RINGS
}

\author{
P. M. COHN \\ To the memory of TADASI NAKayama
}

\section{Introduction}

Many questions about free ideal rings ( = firs, cf. [5] and $\S 2$ below) which at present seem difficult become much easier when one restricts attention to local rings. One is then dealing with hereditary local rings, and any such ring is in fact a fir $(\S 2)$. Our object thus is to describe hereditary local rings. The results on firs in [5] show that such a ring must be a unique factorization domain; in $\S 3$ we prove that it must also be rigid (cf. the definition in [3] and $\S 3$ below). More precisely, for a semifir ${ }^{1)} R$ with prime factorization rigidity is necessary and sufficient for $R$ to be a local ring.

$\S 4$ gives an example of a right fir (in fact a principal right ideal domain) with prime factorization, which is not left hereditary and hence is not a left fir. Since the example is of a local ring, this provides an example of a rigid unique factorization domain which is a semifir but not a fir.

The final section concerns the centre of a hereditary local ring. If this is not a field, then both the ring and its centre are discrete valuation rings. This improves a result of Northcott [8] who showed that the centre, if not a field, must be a 1-dimensional regular local ring. The actual result proved in $\S 5$ is rather more general (apart from the stronger conclusion) in that the hypothesis is weaker: we do not require the existence of a central non-unit $(\neq 0)$ but merely a 'large' non-unit, and in an integral domain every central non-unit $\neq 0$ is large.

\section{Hereditary and semihereditary local rings}

Throughout, all rings are associative with 1 , and all modules are unital. We recall that a ring $R$ is said to be $p$-trivial ( = projective-trivial) if there is

Received May 27, 1965.

1) Semifirs, called 'local firs' in [5] have been renamed here (by analogy with 'semihereditary') to avoid confusion with local rings. 
a projective $R$-module $P$ such that each finitely generated projective $R$-module has the form $P^{n}$ for a unique integer $n$. In particular, such a ring has invariant basis number (i.e. any two bases of a free $R$-module have the same number of elements). An integral domain (not necessarily commutative) with invariant basis number, in which all (finitely generated) right ideals are free, is called a right (semi) fir ; ${ }^{11}$ left (semi) firs are defined similarly. In [5] it was shown that every right semifir is a left semifir, so we may simply speak of semifirs. By a local ring we understand as usual a ring in which the sum of two nonunits is a non-unit.

Since a local ring has a skew field as homomorphic image, it certainly has invariant basis number. Further, all projectives are free ${ }^{21}$ and this shows that a local ring must be $p$-trivial. Now it was proved in [6] (Theorem 2) that any right (or left) semihereditary $p$-trivial ring $R$ is a total matrix ring over a semifir, $R \cong T_{n}$ say. If $n>1$, the equation $1=e_{11}+\left(1-e_{11}\right)$ in $R$ contradicts the fact that $R$ is local; hence $n=1$ and $R \cong T$ is itself a semifir. Similarly, if $R$ is right hereditary (and local) it is a right fir ([6], Theorem 1 ) so we obtain

THEOREM 1. A right or left semihereditary local ring is a semifir; a right hereditary local ring is a right fir.

In particular this shows that a right hereditary local ring is an integral domain. We recall from [5] that a right fir satisfies the ascending chain condition for principal right ideals and hence that every non-unit has a left factor which is a prime ([5], proof of Theorem 2.8). Moreover, by Theorem 2.8 of [5], every left and right hereditary local ring is a unique factorization domain, (UFD) in the sense of [4]. We return to this result in the next section.

\section{Rigid unique factorization domains}

We begin with an obvious general remark.

Lemma 1. Let $R$ be a semihereditary local ring. Given $a, b \in R$ such that $a R \cap b R \neq 0$, then $b R \subseteq a R$ or $a R \subseteq b R$.

2) This is only needed here for finitely generated projectives. The general result (projectives over a local ring are free) was first explicitly proved by Kaplansky [7], but it may be worth noting that this is an immediate consequence of Azumaya's form of the Krull-Schmidt theorem [1]. 
Proof. By Theorem $1, R$ is a semifir and hence $a R+b R=d R$, for some $d \in R$. This means that $a=d a_{0}, b=d b_{0}$ and $a_{0} u-b_{0} v=1$. Since $R$ is a local ring, $a_{0}$ or $b_{0}$ must be a unit, and accordingly, $b R \subseteq a R$ or $a R \subseteq b R$.

If $R$ is a left and right fir, then $R$ is a UFD, by Theorem 2.8 of [5]. More generally, this holds for a semifir in which each non-zero element not a unit can be written as a product of primes. The next theorem gives a criterion for such a ring to be local. We recall the following definition:

A (not necessarily commutative) UFD $R$ is said to be rigid if, for any two prime factorizations of an element $a$ of $R$ :

$$
a=p_{1} p_{2} \cdots p_{r}=q_{1} q_{2} \cdots q_{r}
$$

there exist units $u_{0}, u_{1}, \ldots, u_{r}\left(u_{0}=u_{r}=1\right)$ such that

$$
q_{i}=u_{i-1}^{-1} p_{i} u_{i}(i=12 \cdots r)
$$

THEOREM 2. Let $R$ be a semifir with prime factorization (and hence a UFD). Then $R$ is rigid if and only if it is a local ring.

By the preceding remarks the theorem applies in particular to rings which are left and right firs.

Proof. Let $R$ be a local ring and consider any two prime factorizations (1) of an element $a$. Then $p_{1} R \cap q_{1} R \neq 0$; since $p_{1}$ is a non-unit and $q_{1}$ a prime, we deduce from Lemma 1 that $p_{1} R \subseteq q_{1} R$, and by symmetry, $q_{1} R \subseteq p_{1} R$. Hence $p_{1} R=q_{1} R$, i.e. $q_{1}=p_{1} u_{1}$ for some unit $u_{1} \in R$. Cancelling $p_{1}$ in (1) we obtain

$$
p_{2} \cdots p_{r}=u_{1} q_{2} \cdots q_{r}
$$

and induction on $r$ shows $R$ to be rigid.

Conversely, let $R$ be rigid; we shall show that $R$ is a local ring by showing that the sum of a non-unit is a unit. Let $a$ be a non-unit and $u$ a unit; since $R$ is a UFD we can write $a=p a_{1}$ where $p$ is a prime. Now we have the identity

$$
\left(1+p a_{1}\right) p=p\left(1+a_{1} p\right) .
$$

If $1+p a_{1}$ were a non-unit, then by rigidity $1+p a_{1}=p b$ for some $b \in R$, whence $1=p\left(b-a_{1}\right)$, which contradicts the fact that $p$ is a non-unit. Hence $1+a$ is a unit, as we wished to show. 
Combining this result with Theorem 1 , we obtain the

Corollary. A (left or right) semihereditary local ring with prime factorization (and in particular, a left and right hereditary local ring) is a rigid UFD.

\section{A principal right ideal domain with prime factorization which is not left hereditary}

Let $R$ be a UFD with invariant basis number, which is not a field, and consider $R^{N}$, the direct product of a countable number of copies of $R$. We propose to show that $R^{N}$, as right $R$-module, cannot be projective. Put $A^{(i)} \cong R$, $A_{n}=\prod_{n+1}^{\infty} A^{(i)}$, then $A_{n} \cong R^{N}$ for $n=1,2 \cdots$. If $R^{N}$ were projective, we would have an equation

$$
A_{1} \oplus B=\sum_{I} \oplus C_{i} \quad\left(C_{i} \cong R\right)
$$

where $i$ runs over some index set $I$. Denote by $f_{i}$ the projection onto $C_{i}$ and take $a \in R$, where $a$ is not zero or a unit. Then $\bigcap_{n} R a^{n}=0$ and hence $\bigcap_{n} C_{i} a^{n}$ $=0$ for each $i \in I$. It follows by a theorem of Chase [2] that there exist integers $m, n$ such that

$f_{i}\left(A_{n} a^{m}\right)=0$ for all but a finite number of $i \in I$.

Since $f_{i}\left(A_{n} a^{m}\right)=f_{i}\left(A_{n}\right) a^{m}$ and $a^{m}$ annihilates no element of $C_{i}$, we have

$$
f_{i}\left(A_{n}\right)=0
$$

for all but a finite number of $i \in I$. Suppose that (3) holds except when $i=i_{1}, \ldots, i_{k}$ and write $C^{\prime}=\sum_{1}^{k} C_{i}, C^{\prime \prime}=\sum_{j \neq i \nu} C_{j}$ then (2) may be rewritten as

$$
A^{(1)} \oplus \cdots \oplus A^{(n-1)} \oplus A_{n} \oplus B=C^{\prime} \oplus C^{\prime \prime}
$$

now (3) shows that $A_{n} \subseteq C^{\prime}$ and it follows that $A_{n}$ is complemented in $C^{\prime}$, i.e. there exists $D$ such that

$$
A_{n} \oplus D=C^{\prime}
$$

Since $A_{n} \cong R^{N}$, we have $R \oplus A_{n} \cong A_{n}$. Adding $R$ to (4) and remembering that $C^{\prime} \cong R^{k}$, we obtain

$$
R^{k+1} \cong R^{k}
$$

which is impossible in a ring with invariant basis number. Thus we have proved 
Theorem 3. If $R$ is any UFD with invariant basis number which is not a field, then $R^{N}$ is not projective.

COROLLARY. If $R$ is a semifir with prime factorization then $R^{N}$ is not projective.

Let $k$ be any field with an endomorphism $\sigma$ which is not surjective ${ }^{31}$, and consider the ring $R=k[[x ; \sigma]]$ of all skew power series, consisting of all infinite series

$$
\sum x^{n} \alpha_{n} \quad\left(\alpha_{n} \in k\right),
$$

with multiplication according to the rule

$$
\alpha x=x \alpha^{\circ} .
$$

The mapping $\sigma$ can be extended to an endomorphism of $R$ by defining $x^{\sigma}=x$.

It is clear that $R$ is a local ring with maximal ideal $x R$ and is a principal right ideal domain (in fact $R$ is a discrete valuation ring in the sense defined below, $\S 5$ ) ; moreover, $R$ has prime factorization and so is a rigid UFD. All these facts are easily verified; we shall now show that $R$ is not a left fir, thus in $R$ we have an example of a one-sided fir.

To show that $R$ is not a left fir we must find a left ideal which is not free; now by Theorem 3, Cor., $R^{N}$ is not projective, so it is enough to find a left ideal isomorphic to $R^{N}$. To obtain such an ideal we need only take a sequence of elements $\left(u_{n}\right)$ say, tending to zero, which is left $R$-free. For then $\sum a_{n} u_{n} \in R$ for all $\left(a_{n}\right) \in R^{N}$ and by freeness, the mapping

$$
\left(a_{n}\right) \rightarrow \sum a_{n} u_{n}
$$

is an isomorphism of left $R$-modules. Let $\rho$ belong to $k$ but not to $k^{\sigma}$. Then we assert that the sequence $\left(x \rho x^{n}\right)$ has the required properties. Clearly it tends to zero, and if

$$
\sum c_{i} x \rho x^{i}=0
$$

then by cancelling a power of $x$, if possible, we may assume that $c_{0} \neq 0$. Then

$$
\sum x^{i+1} c_{i}^{i+1} \rho^{\sigma^{i}}=0
$$

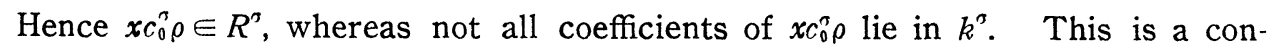

3) It must be injective, since $1^{\sigma}=1$ by definition. 
tradiction, and it shows that the elements $x \rho x^{n}$ are left $R$-free, as asserted.

\section{The centre of a hereditary local ring}

In [4] it was shown that a rigid UFD which is commutative is necessarily a discrete valuation ring. In general we define a discrete valuation ring (DVR) as an integral domain $R$ ( $\cot$ necessarily commutative) with a prime $p$ such that every non-zero element of $R$ is of the form

$$
p^{r} u(r \geq 0, u \text { a unit }) \text {. }
$$

When $R$ is commutative, this reduces to the usual definition. A general DVR is clearly a rigid UFD and it may be verified that a rigid UFD is a DVR if and only if any two primes are right associated.

An element $a$ of an arbitrary ring $R$ is said to be large if $a R$ is a large right ideal in $R$, i.e. if

$$
a R \cap c \neq 0 \text { for all non-zero right ideals } c .
$$

It is easily verified that in any integral domain the set $S$ of large elements is closed under multiplication and left division. Moreover, in a semifir (or more generally in any weak Bezout ring) the set $S$ of large elements satisfies the generalized Ore condition, hence in this case the ring $R$ admits a ring of right fractions with respect to $S, R S^{-1}$ say, and this ring is again a semifir. We shall omit the (easy) proofs as we do not need these results. In fact, to adjoin inverses of large elements would be a retrograde step, as the next theorem shows.

THEOREM 4. Let $R$ be a (left or right) semihereditary local ring with prime factorization. Then $R$ is a discrete valuation ring if and only if it contains $a$ large non-unit.

Proof. Let $R$ be a left or right semihereditary local ring with prime factorization. Then by Theorem $2, R$ is a rigid UFD. Now assume that $R$ contains a large non-unit $c$, say. Given any prime $p \in R$, we have $c R \cap p R \neq 0$ and hence $c=p c^{\prime}$. Write

$$
c=p^{k} u
$$

where $k \geq 1$ is chosen maximal (clearly $k$ is bounded by the number of factors in a prime decomposition of $c$ ). Then $u$ must be a unit, for by $(5), p$ is large 
and so $\not R \cap u R \neq 0$; if $u$ were not a unit, this would mean (by Lemma 1) that $u=p u^{\prime}$, which, inserted in (5), contradicts the maximality of $k$. The argument applies to all primes of $R$; it shows that all primes are large and hence are right associated. Therefore $R$ is a discrete valuation ring. Conversely, in a DVR, the unique prime is a large non-unit.

The result applies in particular to non-zero centre elements (which in an integral domain are always large), to show that a left and right hereditary local ring with centre not a field is a DVR. But now it is no longer necessary to assume that the ring is a UFD. The precise result is

TheOREM 5. Let $R$ be a right hereditary local ring whose centre is not a field. Then $R$ is a discrete valuation ring, and so is its centre.

Proof. Let $c$ be a central element which is not zero or a unit. As before, we can show that for any prime $p$, we have $c=p c^{\prime}$. Suppose that for every $n$ there exists $u_{n} \in R$ such that

$$
c=p^{n} u_{n}
$$

Since $c$ is central, $p^{n} u_{n}=u_{n} p^{n}$ and we have the strictly ascending sequence of right ideals

$$
c R=u_{0} R \subset u_{1} R \subset \cdots
$$

But by Theorem $1, R$ is a right fir, and this satisfies the ascending chain condition for principal right ideals. Hence (6) cannot hold for all $n$, and this means that we can again choose $k$ in $(5)$ to be maximal. Then $u$ must be a unit, and as before it follows that all primes are right associated. To complete the proof we need only show that every non-unit $\neq 0$ has a prime factorization. Let $a \in R$ and suppose that for each $n$ there exists $a_{n}$ such that

$$
a=p^{n} a_{n} .
$$

Taking $n=k r$ where $k$ is as in (5) and $r$ is arbitrary, we have

$$
\boldsymbol{a}=\boldsymbol{p}^{k r} \boldsymbol{a}_{k r}=\left(c u^{-1}\right)^{r} a_{k r}=c^{r} u^{-r} a_{k r}=u^{-r} a_{k r} c^{r},
$$

hence

$$
a R \subset u^{-1} a_{k} R \subset u^{-2} a_{2 k} R \subset \cdots
$$

which again gives a contradiction. This shows $R$ to be a DVR. If we now choose $c$, in the centre of $R$, so that $k$ in (5) takes its least positive value, then 
it is easily seen that every centre element has the form $c^{n} v$, where $v$ is a unit, again in the centre. Hence the centre of $R$ is also a DVR, and the proof is complete.

\section{REFERENCES}

[1] G. Azumaya, Corrections and supplementaries to my paper concerning Krull-RemakSchmidt's theorem, Nagoya Math. J. 1 (1950), 117-124.

[2] S. U. Chase, On direct sums and products of modules, Pacific J. Math. 12 (1962), $847-854$.

[3] P. M. Cohn, Factorization in non-commutative power series rings, Proc. Cambridge Phil. Soc. 58 (1962), 452-464.

[4] P. M. Cohn, Noncommutative unique factorization domains, Trans. Amer. Math. Soc. 109 (1963), 313-331.

[5] P. M. Cohn, Free ideal rings, J. Algebra 1 (1964), 47-69.

[6] P. M. Cohn, A remark on total matrix rings over firs, Proc. Cambridge Phil. Soc. 62 (1966) $1-4$.

[7] I. Kaplansky, Projective modules, Ann. of Math. 68 (1958), 372-377.

[8] D. G. Northcott, The centre of a hereditary local ring, Proc. Glasgow Math. Association 5 (1962), 101-102.

Queen Mary College,

University of London. 